\title{
Korruption, Ämterkauf und Patronage in Florenz. Informelle Politik im italienischen Stadtstaat und der Toskana
}

\section{Clemens Steinwender}

Kerngebiet: Neuzeit

eingereicht bei: Dr. Grüne Niels, MA

eingereicht im: SS 2014

Rubrik: PS-Arbeit

\begin{abstract}
Corruption, Purchase of Administrative Offices and Patronage in Florence. Informal Politics in the Italian City State and Tuscany

This proseminar-paper deals with the corruption and patronage in Late Medieval and Early Modern Florence. The reign of the Medici is especially looked at considering prominent figures such as Lorenzo de Medici or Cosimo de Medici. The necessary institutions for this to happen are adressed as well as the forms these practices had. The ties with the pope and the church are also part of the paper. As will be shown, the leaders of Florence often had to tolerate forms of corruption due to the nature of this Italian city state.
\end{abstract}

\section{Einleitung}

"Die Alleinherrschaft wird leicht zur Tyrannis, die Herrschaft einer bevorrechtigten Schicht mit Leichtigkeit zur Oligarchie, und die Demokratie artet unschwer zur Anarchie aus. Führt also der Gründer eines Staatswesens eine dieser drei Regierungsformen ein, so ist es nur für kurze Zeit. Es lässt sich durch kein irdisches Mittel verhindern, daß sie in ihr Gegenteil ausartet; denn Gut und Schlecht sind einander in diesem Fall sehr ähnlich."

1 Niccolò Machiavelli, Gedanken über Politik und Staatsführung, übs. von Rudolf Zorn, Leipzig 1941, S. 9. 
Dies ist ein Zitat aus einem Werk von Niccolò Machiavelli, in dem politische Mittel und die Definition des Politischen an sich thematisiert werden. Er vertritt die These, dass keine Machtstruktur für immer nur Gutes hervorbringen kann, sondern das Schlechte unweigerlich auf dem Fuße folgt. Eine der Ursachen für so eine Entwicklung könnte man in dem Phänomen der Korruption sehen. Doch was man heutzutage unter diesem Begriff versteht, muss nicht zwangsweise der gleichen Bedeutung entsprechen wie im Italien der Renaissance.

Es kann im Allgemeinen ein Zusammenhang zwischen Machtmissbrauch und Illegalem hergestellt werden, sei dies nun auf moralischer oder politischer Ebene. Die Forschung weist in diesem Feld allerdings noch Lücken auf. So fehlen etwa weitreichende Studien über Handelskorruption oder die Verwicklung der Kirche in Bestechungen und andere unlautere Praktiken. Private Dokumente, Berufungsblätter und auch politische Schriften können wertvolle Einblicke in dieses Themenfeld bieten. Herrschaften in Verbindung von geistlicher und weltlicher Macht sind dabei ein besonders lehrreiches Beispiel dieser Praktiken, wobei vor allem die italienischen Stadtstaaten aufgrund ihrer urbanen Gesichtspunkte hervorstechen. Einer dieser Stadtstaaten, namentlich Florenz, steht im Zentrum dieser Arbeit. ${ }^{2}$

Die Forschungsfrage, auf die sich der Autor konzentrieren will, ist folgende: Mussten die Herren der Stadt Florenz, später die Medici und der Großherzog, Korruption in einem gewissen Maße tolerieren? Wie stark wirkte sich die informelle Politik auf die Regierung der Stadt und der Toskana aus? Die Hypothese des Autors besagt, dass es die Herrschaftsstruktur des Stadtstaates Florenz und auch der Toskana unabdingbar machte, Korruption zumindest in kleinem Ausmaß tolerieren zu müssen. Dabei spielte auch die informelle Politik eine nicht zu unterschätzende Rolle. Um diese Fragen ausreichend beantworten zu können und die aufgestellte These zu testen, wird eine Reihe von wissenschaftlichen Schriften zu Rate gezogen, die sich schon diesem Thema gewidmet haben. Zum einen wäre Jean-Claude Waquet zu nennen, der sich in seinem Aufsatz "Some considerations on corruption, politics and society in sixteenth and seventeenth century Italy"3 mit der politischen Dimension im sechzehnten und siebzehnten Jahrhundert beschäftigt. Moritz Isenmann stellt sich der Frage der Korruption in diesem Teil Italiens zur Zeit des späten Mittelalters. In dem Aufsatz "Rector est Raptor. Korruption und ihre Bekämpfung in den italienischen Kommunen des späten Mittelalters“4 greift er dieses Thema in einem Umfeld auf, das noch vor der Machtergreifung der Medici datiert und somit einen Überblick über die Anfänge und die Struktur dieses Phänomens bietet. Sollte man Interesse an den Verwicklungen der Medici mit der katholischen Kirche

2 Guy Geltner/Maaike von Berkel, Fighting Corruption in Premodernity, East and West: A Literature Review, S. 2 ff., [http://anticorrp.eu/publications/corruption-in-premodernity-east-and-west-a-literature-review], eingesehen 29.05.2014,

3 Jean-Claude Waquet, Some considerations on corruption, politics and society in sixteenth and seventeenth century Italy, in: Little, Walter/Carbó, Edward Perader (Hrsg.), Political corruption in Europe and Latin America, New York 1996, S. 21-40.

4 Moritz Isenmann, Rector est Raptor. Korruption und ihre Bekämpfung in den italienischen Kommunen des späten Mittelalters, in: Karsten, Arne/Thiessen, Hillard von (Hrsg.), Nützliche Netzwerke und korrupte Seilschaften, Göttingen 2006, S. 208-230. 
hegen, so ist die Monographie von Christian Wieland, „Fürsten, Freunde, Diplomaten. Die römisch-florentinischen Beziehungen unter Paul V. (1605-1621)“5, zu empfehlen. In ihr behandelt Wieland eine Vielzahl von Praktiken und historischen Begebenheiten, die einen Aufschluss über informelle Politik und Korruption in diesem Themenbereich geben können.

Die Analyse beginnt mit einer kurzen historischen Einleitung über die Stadt Florenz und die Toskana. Der Aufstieg der Medici und die Entwicklung hin zu einem Großherzogtum sind Thema dieses Kapitels. Darauf folgt eine Betrachtung der spätmittelalterlichen Verhältnisse und des Überganges zur Herrschaft der Medici. Im Anschluss daran wird die weitere Entwicklung in den folgenden Jahrhunderten skizziert, wobei hier schon ein Wandel der Machtverhältnisse zu beobachten ist. Danach werden die Verwicklungen und diplomatischen Beziehungen der Familie Medici mit der päpstlichen Kanzlei und den religiösen Institutionen thematisiert. Im letzten Kapitel werden die Primärquellen von Niccolò Machiavelli im Hinblick auf Korruption und informelle Politik betrachtet.

\section{Der Stadtstaat Florenz und die Medici}

Eine Zäsur in der Geschichte von Florenz waren die Pestwellen von 1363. Durch diese kam es zu großen Umwälzungen, aus denen die wirtschaftlichen Eliten der Bankiers und Textilproduzenten als Sieger hervorgingen. Ein schmaler Zirkel aus bis zu fünf Dutzend Personen hatte die Kontrolle über die Politik bis in das fünfzehnte Jahrhundert hinein inne, als eine neue Machtstruktur aufkam. Der Versuch der Familie Visconti aus Mailand, sich in der Stadt zu etablieren, schlug fehl. Die oligarchischen Machthaber lernten, die Masse ruhig zu halten und ihre Position zu sichern. Dies führte jedoch zu zahlreichen Vorwürfen der moralischen Dekadenz und der Konzentration auf den eigenen Vorteil. Aus den verschiedenen politischen Strömungen heraus bildeten sich schlussendlich zwei Lager, die um die Herrschaft rangen. ${ }^{6}$

Cosimo di Giovanni di Bicci de' Medici war der Anführer eines Lagers und schaffte es, durch geschickte Propaganda an die Macht zu gelangen. Die Medici hatten ihre Machtbasis durch Gewalttätigkeit und Aufstände geschaffen. Ein eigenes Bankenunternehmen verschaffte innen ein wirtschaftliches Standbein sowie ein stabiles Familienvermögen. Besonders durch Verbindungen mit Rom und der Kurie wurden große Gewinne erzielt. Geschenke, Patronage und Unterstützung verhalfen der Familie zu einem loyalen Netzwerk an adeligen Familien. Ab 1425 wurde der Kampf um die Herrschaft verdeckt ausgetragen, bevor er härtere Züge annahm. Ein Verbannungs- und Diskreditierungsversuch gegen die Medici endete jedoch mit der Stärkung der Position dieser Familie. Bei einer Wahl, in der die Medici zahlreicher waren als ihre Gegenspieler, wurde der Konflikt entschieden. Die Kontrahenten der Familie wurden ausgemerzt und die Macht über Florenz an sich gerissen. Obwohl es zuerst einigen Widerstand gab, wurde dieser im zweiten Staatsstreich

5 Christian Wieland, Fürsten, Freunde, Diplomaten. Die römisch-florentinischen Beziehungen unter Paul V. (1605-1621) (Norm und Struktur. Studien zum sozialen Wandel im Mittelalter und der frühen Neuzeit 20), Köln 2004

6 Volker Reinhardt, Geschichte von Florenz, München 2013, S. 51-54. 
von 1458 endgültig beiseite gefegt. Militärische Erfolge gegen Mailand und die finanzielle Unterstützung der Sforza sorgten für einen stabilen Verbündeten. ${ }^{7}$

Den Höhepunkt der Macht der Medici stellte die Herrschaft von Lorenzo il magnifico dar. Er entkam einem Attentat im Jahre 1478, bei dem sein Bruder das Leben verlor. Dies führte zu einer rigorosen Verfolgung der Verschwörer, nach der die Medici die Zügel noch straffer anzogen. Der "erste Mann der Republik“ konnte die notorisch unruhige Stadt nur schwer unter Kontrolle halten. Piero de Medici verspielte um das Jahr 1494 herum sehr viel Kapital und Ansehen seiner Familie, was zu einem Verfall der Machtposition führte. Endgültig gebrochen wurde die Herrschaft der Medici durch den Einfall Frankreichs, dessen König die Familie aus der Stadt verbannte. Sie fand in Rom Zuflucht, wo sich noch Verwandte aufhielten. Als Giovanni de' Medici 1513 zum Papst gewählt wurde, konnte die Familie erneut eine Vormachtstellung etablieren. Als der zweite Papst aus der Familie, Giulio de' Medici, gewählt wurde, fand der sacco di romå statt, woraufhin die Familie erneut vertrieben wurde. ${ }^{9}$

Doch selbst nach solch katastrophalen Rückschlägen konnten die Medici immer wieder zurückkehren. Cosimo der Jüngere stieg aus einer Seitenlinie auf und eroberte Siena und die Südtoskana, nachdem er die politische Macht in Florenz wieder ergriffen hatte. Nach diesen Leistungen wurde er zum Herzog ernannt und die Toskana zum Großherzogtum ausgerufen. In der Mitte des sechzehnten Jahrhunderts standen die Zeichen in Florenz und der Toskana auf Stabilität. ${ }^{10}$

\section{Informelle Politik und Korruption im Spätmittelalter und der Anfangszeit der Medici}

Im Spätmittelalter trug der Begriff Korruption eine andere Bedeutung als die, die wir heute kennen. So wurde diese etwa Abusus (Missbrauch), Mala Consuetudo (schlechte Gewohnheit), Defectus oder Scandalum genannt. Sie bezeichnete im weitesten Sinne den Verfall von materiellen und immateriellen Dingen, darunter fielen auch Delikte und Missstände. Der Verfall des Menschen und der Moral war ein zentrales Thema. Die Baratteria (Amtsmissbrauch) schien dabei sehr häufig auf, wobei sie sicherlich auch dazu benutzt wurde, politische Gegner anzugreifen. Zeugnisse dieser Praktiken fanden sich unter anderem in Dantes Werken oder auch bei Orfino da Lodi, der in seiner Abhandlung "De regimine et sapientia potestatis" die Korrumpierbarkeit der Richter angriff und ihre Schwächen aufzeigte. Zu diesen gehörten unrechtmäßige Bereicherung und die Verzögerung von Rechtsstreitigkeiten. In der Ikonographie jener Zeit wurde dies ebenfalls offenbart, so befand sich etwa in der Scrovegni-Kapelle das Bild eines Magistrats mit den Krallen eines Raubvogels. ${ }^{11}$

7 Reinhardt, Geschichte Florenz, S. 5-73.

8 Die Plünderung Roms durch Landsknechte und Söldner, unter Befehl von Karl V.

9 Reinhardt, Geschichte Florenz, S. 74-97.

10 Ebd., S. 98-101

11 Isenmann, Rector est Raptor, S. 209 ff. 
Die Baratteria an sich beinhaltete auch die Begriffe Unterschlagung und Erpressung, in erster Linie wurde darunter aber Bestechlichkeit verstanden. Die Gerechtigkeit wurde so zum Tauschhandel (baratto). Durch das Spesenwesen litt vor allem die Bevölkerung des Umlandes unter dieser Praxis, denn die Magistrate konnten so weitere Gelder für sich selbst eintreiben, was in Konflikt mit ihrer Aufgabe stand. Bernhard von Siena machte seinem Ärger 1425 vor der Kirche Santa Croce Luft, indem er die Magistrate mit gefräßigen Heuschrecken verglich und so verunglimpfte. Es waren jedoch die Glossatoren, welche an dieser Praxis und ihren Folgen am meisten Kritik übten. Dies zeigte sich daran, dass die Baratteria später zu den Majestätsverbrechen gezählt und streng bestraft wurde. ${ }^{12}$

Die Familie und ihre Bande waren in Florenz so wichtig wie in allen Kommunen Italiens. Allerdings setzte sich im Beamtentum ein völlig anderes Selbstverständnis durch. So waren etwa für die verfügbaren Posten nur Qualifikation und Ehre als entscheidend anzusehen, nicht aber die Beziehungen der Familie. Dieses System war seit dem elften Jahrhundert entstanden, da man gegen Macht- und Herrschaftskonzentration ein stetes Misstrauen hegte. Durch das Recht wurden auch Machthaber gebunden, was zum Selbstverständnis der Stadtstaaten beitrug. Mittels komplizierter Wahlverfahren wollte man Manipulationsversuche verringern. Die Verwaltung der Justiz übernahmen Kräfte von außerhalb der Stadt, um die Einflussnahme auf sie einzudämmen. Diese Podestà war auf ein halbes Jahr beschränkt, nach dessen Ablauf der Amtsträger mit seinem Gefolge weiterzog. 13

Trotz all dieser Schutzmechanismen konnte man einen Missbrauch der Amtsgewalt und Einflussnahme auf Amtsträger feststellen. Einer dieser Versuche war die Verteilung von Geschenken. Zu Anfang wurden Gaben von Speisen und Wein noch geduldet, später allerdings unter Strafe gestellt. Aus den Florentiner Statuten von 1325 ist dies deutlich herauszulesen. Es gab allerdings noch andere Versuche der Einflussnahme. 1298 etwa war ein Podestà mit dem Hintergedanken gewählt worden, sich seiner zu bedienen. Nach einiger Zeit wurden die Bürger jedoch dieses Systems überdrüssig und verjagten inn. Weitere Prozesse gegen korrupte Richter konnten beobachtet werden, wobei diese auch als politisches Mittel des Machtkampfes von den führenden Familien eingesetzt wurden. Denn trotz des Systems in Florenz waren einige wenige dominante Familien die Machthaber, die sich der klientelähnlichen Abhängigkeit bedienten. Der starke Mann war der Versuch, sich solchen Erscheinungen zu widersetzen, dafür öffnete man aber der Patronage und dem Klientelismus Tür und Tor. Florenz musste als ein Sonderfall betrachtet werden, denn obwohl die Stadt offiziell eine Republik war, trachteten viele nach einer Kontrolle der Wahlmechanismen und so einer Einflussnahme auf die Stadtpolitik. Demgegenüber stand der Versuch, mithilfe von Gesetzen und der Podestà die Ausbreitung der Ämterpatronage und des Klientelismus einzuschränken. ${ }^{14}$

Mit dem Aufstieg der Medici zeigte sich der Einfluss der informellen Politik sehr deutlich. So lautete der Leitspruch der Familie „Freunde durch Geld”, was bezeichnend für ihre

12 Isenmann, Rector est Raptor, S. 212-215.

13 Ebd., S. 215-219.

14 Ebd., S. 214-224. 
Strategie war. Gefolgsleute wurden aus den arti maggiori mithilfe von Geld, Protektion, Verschwägerung und durch Vergabe kleiner Aufträge geschaffen: „Aus seinen schier unerschöpflichen Reserven bezahlte Cosimo Steuerschulden, lieferte Mitgiften, machte Patengeschenke und steuerte das Startkapital für Geschäftsgründungen bei."15 Die Empfänger solcher Gaben wussten, was sie ihrem Patron schuldeten, nämlich die Gewährleistung des Wohls der Medici in ihren Ämtern. ${ }^{16}$

Die Familie konnte ihre Machtposition auch deshalb aufbauen, weil sie neue Dinge wagte und in die Wege leitete. So kam es etwa zu einer Manipulation der Wahlbeutel, aus denen man die Kandidaten für ein Amt zog. Sie wurden ausgetauscht und großteils mit den Namen von Parteigängern gefüllt. Das war außerordentlich: „Die Manipulation der Wahlbeutel im Herbst 1434 war eine Revolution. " ${ }^{17}$ Dies stellten die Medici sicher, indem das Amt des Befüllers der Beutel direkt mit ihren loyalsten Gefolgsleuten besetzt wurde. Vermutlich kamen bereits Zeitgenossen diesem Trick bald auf die Schliche, trotzdem fand diese Praxis keine nennenswerten Ankläger. Selbst in den Annalen eines Leonardo Bruni fanden diese Methoden keinen Niederschlag. ${ }^{18}$

In der Person des Lorenzo il magnifico verbarg sich einer der geschicktesten Spieler der politischen Bühne. So nutzte er etwa den Anschlag auf sich und seinen Bruder als Gelegenheit, denn das Attentat wurde als Majestätsverbrechen angesehen und mit der damnatio memoriae bestraft. Er knüpfte ein umfassendes Netzwerk von Kontakten und Klienten, in dem ständig Gefallen eingefordert und neu verteilt werden mussten. Seine Rolle als Schlichter bei Streitigkeiten war begehrt und führte zu weiteren Möglichkeiten. Auch zu Spionagezwecken zog er seine Kontakte heran. Ebenso hatte Lorenzo die Angewohnheit, Söldnerführer anzuheuern und zu deren Vorteil zu intervenieren, was inm weitere Abhängigkeiten verschaffte. Zu seinen Klientelen gehörten Bischöfe, Juristen, Kardinäle, Beamte und Höflinge. Die niedriger stehenden Schichten der Gesellschaft baten um seine Gunst, um dem Gefängnis zu entgehen oder damit er zu ihrem Vorteil in Streitfälle einschritt. Selbst Hochzeiten bedurften des Einverständnisses der grauen Eminenz. ${ }^{19}$

Nachdem die Kassen der Medici aufgrund einer Reihe von kriegerischen Auseinandersetzungen erschöpft waren, berichteten zeitgenössische Quellen davon, dass sich Lorenzo mithilfe von Handlangern aus der Staatskasse bediente, um seine Reserven wieder aufzustocken. Die Schätzungen über die Summe des entwendeten Geldes schwankten, beliefen sich aber meist auf 50.000 bis 200.000 Fiorentini. Lorenzo selbst schien dies ohne Gewissensbisse zu tun, da er sich einredete, es wäre das gute Recht der Medici, sich dieser Ressource zu bedienen, da seine Familie schon so viel für die Stadt getan hätte. Von Diebstahl wollte er nichts wissen. ${ }^{20}$ Selbst im Spätmittelalter, in dem man noch versucht

15 Reinhardt, Geschichte Florenz, S. 60.

16 Ebd., S. 57-61.

17 Ebd., S. 70.

18 Ebd., S. $70 \mathrm{ff}$.

19 Lauro Martines, Die Verschwörung. Aufstieg und Fall der Medici im Florenz der Renaissance, Darmstadt 2004, S. 223 ff.

20 Martines, Verschwörung S. 226 f. 
hatte, der Korruption und ihrer Auswirkungen Herr zu werden, gab es dieses Phänomen. Begriffe wie die Baratteria und der Missbrauch der Podestà traten oft in den Quellen hervor und ließen einen kleinen Blick auf die Ausweitung dieser Praktiken zu. Die Medici, die sich als die Herren der Stadt Florenz sahen, bedienten sich der informellen Politik sehr stark. Durch Klientelismus und Ämterpatronage versuchte man, sich Günstlinge zu verschaffen und Amtsgeschäfte zum eigenen Vorteil zu beeinflussen. Durch Darlehenszahlungen und Gefallen wurden künstliche Abhängigkeiten geschaffen, deren Wert oftmals entscheidend war. Besonders Lorenzo de Medici bediente sich dieses politischen Mittels und schuf sich ein komplexes und weitreichendes Netzwerk. Die informelle Politik spielte zu seiner Zeit und in seinem Regierungsstil eine große Rolle. Dabei mussten die Medici natürlich auch Korruption unter ihrer Regierung zulassen, denn sie selbst bedienten sich oft und gerne der Bestechlichkeit der Menschen. Dieser Eigenschaften erfreuten sich sicherlich auch ihre Gegner, die ihnen allerdings in dieser Hinsicht nicht das Wasser reichen konnten.

\section{Korruption und informelle Politik vom 16. bis zum 18. Jahrhundert}

Die Ausbreitung der Korruption in dieser Zeit war sehr intransparent. Als eines der wenigen Gebiete war die Toskana besser beleuchtet, auch wenn man versuchte, Hinweise auf korruptes Verhalten aus den Archiven herauszuhalten. Bestimmte Quellen wie die Privatbriefe von Beamten oder Geschäftsbücher von Gemeinden boten hierzu wertvolle Hilfestellung. In diesem Feld zeichnete sich vor allem der italienische Historiker Aldo de Maddalena aus. Eines der aufschlussreichsten Werke waren die Racordi, in der Informationen sehr konkret protokolliert wurden. Politische Verfehlungen, die über die Korruption hinausgingen, wurden ebenso behandelt. Laut der Meinung des Autors würde die Korruption Hand in Hand mit dem Versagen eines politischen Systems gehen. Konsequenzen dieses Versagens waren das Fehlen von Balance, Rivalitäten und Unterdrückung. Jedoch musste man mit diesem Werk sehr vorsichtig sein, denn es war eindeutig subjektiv geprägt. Die Autonomie der Provinz Mailand wurde stark befürwortet und so muss hinterfragt werden, ob die Racordi der Beweis für Korruption in Italien zu dieser Zeit waren, oder nur eine weitere geschickte Nutzung des Vorwurfs für den politischen Machtkampf. ${ }^{21}$

Die Kriterien, anhand derer Korruption erkannt werden konnte, waren im sechzehnten und siebzehnten Jahrhundert weithin bekannt. Denn die Beamten und Richter sollten die Ideale verkörpern und ganz im Geiste der justitia animata handeln, mit all den nötigen Tugenden. ${ }^{22}$ Um Missbrauch zu bekämpfen, wurden verschiedene Gesetze erlassen, so zum Beispiel das Verbot der Annahme von Geschenken. Alleine im Herzogtum Toskana wurde dieses zweimal verabschiedet, in den Jahren 1550 und 1576. Andere Verbote beinhalteten den Diebstahl von verwaltetem Geld und den Zugriff auf öffentliche Gelder für private Zwecke. Dieser Diebstahl beinhaltete aber nicht nur den Faktor Geld, sondern umfasste alles, an dem sich die Richter und Beamten bereichern konnten. So wurde etwa der Begriff Peccata genutzt, um Übergriffe des Dieners eines Königs der angrenzenden 
Länder zu beschreiben. Religiöse Begriffe wurden angewendet, um die Korruption zu verdammen. So wurden diese Verbrechen nicht nur als Sünde bezeichnet, sondern auch als Beweis des moralischen Verfalls und als eine Krankheit des Herzens und der Seele angesehen. Der Nährboden für korrupte Handlungsweisen lag in den Eigenschaften Ehrgeiz, Stolz, Furcht und Hass. Ein weiterer Faktor für die Anwendung solcher Praktiken war die Verwicklung in Patronage- und Klientelnetzwerke, in die viele verstrickt waren und deren Pflichten sie erfüllen mussten. ${ }^{23}$ Trotz all dieser Maßnahmen blühte die Korruption. Rom stellte hier einen Sonderfall dar, da der Nepotismus des Papsttums sehr viel Spielraum für informelle Politik und korrupte Seilschaften bot. Das Großherzogtum der Toskana war auch von solchen Ereignissen betroffen. Die Patriarchen von Florenz, Erben der Republik, hatten die Angewohnheit, sich als Herren der Bürokratie zu sehen. So einen Patriarchen zu bestrafen stellte sich jedoch als schwieriger heraus als erwartet. Denn die Verurteilung eines solchen hätte oft zu Spannungen zwischen den Adeligen und den Herzögen der Medici geführt, weswegen die Gnade zu einem der wichtigsten politischen Mittel der Herrscherfamilie wurde. Überhaupt war dieses Phänomen unter den Medici einzigartig. Die Korruption war verbreitet, die Bestrafung bestenfalls schwierig und die Erzürnung der führenden Schicht beinahe sicher. Die Unterstützung des Magistrats war der Korruption allerdings verwehrt, da die Justiz oft aus Beamten von außerhalb der Stadt bestand. Auch wenn sie nicht immer die Ehrlichkeit in Person verkörperten, waren sie aufgrund dieses Umstandes immerhin weniger mächtig, einflussreich und beinflussbar als ihre Kollegen in anderen Städten. Die Bewohner der Toskana genossen so wenigstens einen gewissen Schutz vor den korrupten Handlungen der Justiz. ${ }^{24}$

Mit der Zeit setzte aber ein Wandel in der Stadt Florenz ein. Als die Staatskammern geleert waren, gab man den Beamten die Schuld, die sich angeblich aus innen bereichert hatten. Dies war ein Beispiel, in dem die Korruption erneut politisch genutzt wurde; es war allerdings nicht ganz aus der Luft gegriffen. Denn die Macht war in manchen Teilen vom Herrscher weg zu seinen Dienern gewandert. Der Begriff der Korruption wandelte sich nun und erfüllte eine Doppelrolle. Sie verschaffte den Beamten Geld und gab die Macht zurück an herrschende Schichten, die sich wieder eine starke Position verschafften. Nun galt die Korruption als einzelne, leider wiederkehrende Verfehlung. Für die niederen Schichten waren der moralische Verfall und die soziale Ächtung eine Abschreckung und dämmte diese ein, gleichzeitig wurde sie im Falle eines individuellen Auftretens als tolerabel angesehen. Eine Doppelrolle des Gesetzes setzte ein, das Übeltäter bestrafte und das Phänomen auf kleinere Delikte eingrenzte. Diese Moralisierung der Korruption führte hin zu einem Paradoxon, da Lügen verbreitet wurden, um zum Schein eine Maske der Ehrlichkeit in der Öffentlichkeit tragen zu können. Hier trat die Fragilität des Staates offen zu Tage. Er konnte nicht alle Korruption auslöschen und bestrafen, während manche seiner Gesetze sie sogar noch verschärfte. Der Staat war denen ausgeliefert, die inm dienten und die diese Stellung schamlos zu ihrem eigenen Vorteil ausnutzten. ${ }^{25}$

23 Waquet, Considerations on corruption, S. 26-32.

24 Ebd., S. 33-37.

25 Jean-Claude Waquet, Corruption. Ethics and power in Florence, 1600-1770, Pennsylvania 1992, S. 190195. 


\section{Die Stadt Florenz und das Pontifikat}

Von Ratgebern und Fürsten ausgehende Netze, die auf Klientelstrukturen basierten, konnten vor der modernen Staatlichkeit als ein Motor für deren Entwicklung angesehen werden. Im Zuge der Bürokratisierung stellten diese informellen Netzwerke eine Verbindung, aber auch einen Gegensatz für diese Entwicklung dar. Am besten eignete sich wohl der Begriff der Verflechtung, um diese Patronage- und Klientelsysteme zu beschreiben. Denn um politisch und wirtschaftlich aufzusteigen, bedurfte es meistens der Hilfe familiärer Kontakte oder der Gefälligkeiten der oberen Schichten. Dies wurde oft mithilfe eines sozialen Netzwerkes lokaler Funktionsträger erreicht, in dem die Broker eine wichtige Funktion innehatten. Man konnte sogar sagen, dass die Patronage und der Aufbau des frühneuzeitlichen Hofes sehr eng miteinander verflochten waren. Es schien dabei auch passend, dass sich der Hof ebenso dem Vorwurf der Dekadenz und Rückwärtsgewandtheit stellen musste. Als Zentrum der Politik und der Machtausübung erlangte der Hof enorme Wichtigkeit, ein enormes Klientelnetzwerk wurde zum Referenzpunkt der Elite Europas und der Diskurs zu Hofe wurde zum politischen Diskurs der frühen Neuzeit. Die Ethik und die Form dieses Ablaufes wurden sehr stark von der Patronage bestimmt. Und fast nirgendwo war dies so offensichtlich wie am Hof der Päpste und bei den Machenschaften ihrer Nepotisten. ${ }^{26}$

Besonders prägend war für die italienischen Stadtstaaten der Gegensatz zwischen den Häusern Valois und Habsburg. Dieser europäische Dualismus hinterließ seine Spuren auch in der Innenpolitik. Die Medici-Monarchie selbst war ein Produkt der habsburgischpäpstlichen Allianz. Der sacco diroma hatte die Machtposition der Habsburger eindrucksvoll unterstrichen. Als Gegenleistung für die Krönung Karls V. sollte der Sohn von Clemens VII. zum Herzog der Toskana ernannt werden. Die Heiratsverbindungen der Medici zeigten ebenso dynastische Züge und auch den Versuch, sich gegen beide Seiten abzusichern. Während die erste Medici-Monarchie noch sehr eingeschränkt war, blühte die zweite regelrecht auf. Gelockerte Partnerwahl und mehr politische Freiheit waren ihr inne. Die Medici verstanden es meisterhaft, sich auch mithilfe der päpstlichen Verbindungen neuen Handlungsspielraum in festgelegten Strukturen zu schaffen. ${ }^{27}$

Die Verbindungen der Medici mit dem päpstlichen Hof waren vielschichtig und oft undurchsichtig. Man verständigte sich über das Zeremonielle und feine Unterschiede in Betitelung und Ansprache konnten zu einem gewaltigen Unterschied im Status führen. So hatten etwa gewisse zeremonielle Elemente nur die Aufgabe, die Position des Papstes zu untermauern, beispielsweise der Fußkuss. Subtile Ehrabstufungen waren gegeben, die sich nicht nur auf simples Nichtbefolgen von Anweisungen beschränkten. So lag etwa nicht nur der Kontakt zwischen Herzogtum und Papsttum diesen Überlegungen zugrunde, sondern auch der Austausch zwischen den etablierten Medici und den Aufsteigern aus den anderen italienischen Familien. Dies zeigte sich einmal besonders in einem Brief des Botschafters Niccolini, der sich beim Kardinalnepoten über die Formulierungen des Großherzogs in seinen Schreiben beschwerte. Einer der bedeutendsten Titel war der des

26 Wieland, Fürsten, Freunde, Diplomaten, S. 15-19.

27 Ebd., S. 100-109. 
Eccellenza. Erteilen oder Verweigern dieses Titels waren ein deutlicher Ausdruck politischsozialen Willens. Das Zeremoniell war so sehr dynamisch und bedeutend, Kleinigkeiten umso gravierender. Die Sprache, sowohl der Worte als auch des Körpers, unterstrichen Status, Rang und Position. Als Beispiel für die weitreichenden Konsequenzen solchen Handelns stand die Streitfrage zwischen Savoyen und den Medici. Die Botschafter der Savoyer weigerten sich so hartnäckig, die Medici mit dem Titel des Prinzen anzureden, dass sie erst davon überzeugt werden mussten, dies zu tun. ${ }^{28}$

Die Verteilung von Geschenken fand auch am Hof des Papstes statt. Der Botschafter von Florenz erhielt zahlreiche Naturalien, die er am corte di roma verteilen musste. Der Empfänger und die Menge des Geschenkes stellten die Variablen einer Prozedur dar, die zum Alltag des politischen Geschehens gehörte. Auf der einen Seite stellten diese Akte den Willen zu Freundschaft und Kontakt dar, andererseits konnten aus solchen Beobachtungen auch die Rangordnungen der toskanischen Überlegungen bezüglich der römischen Familien klar erkennbar hervortreten. So existierte eine Liste aus dem Jahre 1615, in der die genaue Menge an Austern niedergeschrieben war, die an jede Person in Rom verschenkt wurde. Laut dieser Liste standen der Kardinalnepot und sein Zugang zum Papst in den Überlegungen der Medici an oberster Stelle. ${ }^{29}$

Das Zeremoniell an sich trug doch einiges an Konfliktpotential in sich. Es bildete die Verbindung zwischen allem politischen Verhalten. Die Gesten des Zeremoniells konstruierten Handlungsräume und jeder Politiker in Rom war damit konfrontiert. An der Spitze dieses Systems stand der immobile Papst. Wenn die Toskana agierte, stand das Kirchenoberhaupt still. Besonders schwerwiegend war der Konflikt zwischen dem Universalanspruch des Papstes und der vermeintlichen Inferiorität der den Papst stellenden Familien gegenüber den Medici. Dies kam in Schreiben und dem Zeremoniell stark zum Ausdruck. ${ }^{30}$

Wie sehr sich informelle Politik und Korruption auf eine Familie auswirken konnten, zeigte die Krise der Familie Barberini nach dem Tod von Urban VIII. im Jahre 1644. Nach dem Ableben eines Papstes wurden die überlebenden Familienmitglieder häufig kritisch betrachtet. Eine übliche Taktik war es deshalb, sich mithilfe von Geschenken und Lobpreisungen bei den neuen Machthabern gut dastehen zu lassen. Im Fall der Barberini jedoch war der Zorn der Bevölkerung und der Adeligen so gewaltig, dass der neue Papst eine Untersuchung einleiten musste. Dies war eine gefährliche Gratwanderung, denn man wollte eigentlich nicht, dass die Barberini untergingen, stellten sie doch ebenso einen Faktor im persönlichen Treuegeflecht und Klientelismus dar. So hatten sie noch zu Zeiten ihres Papstes viele Abhängige und Klienten in kirchliche Positionen eingesetzt. Aber trotz dieser Maßnahmen sollte die Habgier der Familie ihr Untergang sein. Denn die rücksichtslose Schröpfung des Papsttums in wirtschaftlicher und politischer Hinsicht mithilfe von Patronage und Klientelismus wurde zum stärksten Kritikpunkt ihrer Gegner. Mit dem Tode Urbans VIII. waren alle Maßnahmen der informellen Politik plötzlich verwerflich und

28 Wieland, Fürsten, Freunde, Diplomaten, S. 329-334.

29 Ebd., S. 334 f.

30 Ebd., S. 336 u. 356. 
moralisch fragwürdig geworden. Anklagen wegen Untreue und Bestechlichkeit wurden immer zahlreicher, weswegen die Familie nach Paris floh. Zwar hatte sie sich einen großen Klientelstand aufgebaut, dieser hatte aber verschiedene Machtstellen mit einem Schlag eingebüßt. Die Familie schaffte es, sich aus dieser Misere zu befreien, indem sie sich mit der neuen Papstfamilie aussöhnte und deren Klientelnetzwerke mit den Eigenen verband. Aus dieser Begebenheit heraus waren jedoch die Grenzen der Patronage und des Klientelismus klar zu Tage getreten. Die Vorsorge für die Familie mithilfe des Pontifikats war bei Verlust der Machtposition verwerflich oder gar rechtlich strafbar geworden, was natürlich von den politischen Gegnern stark genutzt wurde. Solche Anschuldigungen kamen allerdings häufig genau dann auf, wenn die Dominanz der Herrschenden ins Wanken geriet. ${ }^{31}$

Hier zeigt sich erneut, wie stark sich die informelle Politik auf die Regierung der Medici und der Toskana auswirkte. Ein guter Kontakt zu den Päpsten war unabdingbar, wollte Politik in großem Stil betrieben werden. Dabei war das Informelle keineswegs zu verachten. Das Zeremoniell zu Hofe war immens bedeutend, selbst kleinere Auffälligkeiten oder Anreden konnten eine versteckte Botschaft beinhalten. Die Krise der Familie Barberini nach dem Tode von Urban VIII. stand stellvertretend für die Gefahren solcher Praktiken, da diese meist mit dem Wegfall der Machtposition von Widersachern zur Kritik genutzt wurden. Es zeigte sich erneut, dass Korruption in gewissem Maße zu tolerieren war, da man sich dieses Phänomens häufig selbst bediente. Es war aber eine Gratwanderung zwischen dem Innehaben der Macht und dem Verlust ebenjener. Die Bedeutung des Informellen kann in dieser Hinsicht nicht genug betont werden.

\section{Niccolò Machiavelli über Korruption und informelle Politik}

Einer der einflussreichsten Theoretiker über Politik und Staat war Niccolò Machiavelli. Auch er hatte sich mit dem Thema der Korruption beschäftigt. So musste sie immer in Zusammenhang mit der "Guten Republik" gesehen werden. Das Höchste in einer Republik war die civic virtue. In keinem machiavellistischen Modell entstand die Korruption systematisch, stattdessen stand ihr eine nüchterne Analyse gegenüber. Er definierte sie wie folgt: Korruption war eine persönliche, individuelle Dekadenz von Bürgern und Herrschern. Die guten Menschen waren frei und arbeiteten für die Republik und für andere, während die Schlechten von Lust, Habgier und Ehrgeiz getrieben wurden. Die bedeutendste Ausprägung der Korruption war laut Machiavelli die Nutzung des normalen Bürgers und der Beamten für die eigenen Zwecke. Herrscher hätten durch Schmeichelei und Beeinflussung leicht Einfluss auf sie nehmen können. Innerhalb korrupter Städte nahmen Adelige oft durch private Wege Einfluss, etwa mit der Hand ihrer Töchter, dem Leihen von Geld oder der Patronage. Auf diese Weise würde ein Prozess in Gang gesetzt, der mit der völligen politischen Ohnmacht der Bürger enden würde. ${ }^{32}$

31 Ulrich Köchli, Nepoten, Pfründen und Klienten. Die Krise der Familie Barberini nach dem Tod Urbans VIII., in: Nützliche Netzwerke und korrupte Seilschaften, hrsg. v. Arne Karsten/Hillard von Thiessen, Göttingen 2006, S. 163-180, hier S. 164-177.

32 S. M. Shumer, Machiavelli: Republican politics and its corruption, in: Political Theory 7 (1979), Heft 1, S. 5-34, hier S. $8 \mathrm{f}$. 
Am Beispiel Florenz erklärte Machiavelli, weshalb Menschen mit persönlichem Ehrgeiz nie das Wohl aller im Sinne haben konnten. Eigennützige Politik hatte die Entscheidungsfindung unterminiert, weshalb Entscheidungen nicht zum Wohle der Stadt getroffen werden konnten. Die Lektüre von Machiavelli lehrte, dass diese Tatsachen Teil des gleichen Symptoms waren. Korrupte Menschen konnten keine wirksamen Maßnahmen gegen Korruption einführen. Dem stellte er die Freiheit gegenüber, mit der auch die politische gemeint war. Obwohl die Freiheit Macht verkörperte, war es keine Macht, um zu beherrschen. Ironischerweise aber war der Konflikt in Freiheit und Korruption genau gleich verachtenswert. Laut Machiavelli bedeutete Korruption, in treffendster Weise die Eigenschaften zu beschreiben, aufgrund derer das Ideal der guten Politik verfehlt wurde. ${ }^{33}$

In den großen Werken von Machiavelli über die Geschichte von Florenz und die politische Theorie ließen sich viele Hinweise auf Korruption und das Informelle in der Politik finden. So beschrieb er Cosimo de' Medici etwa folgendermaßen:

"Cosimo war ein äußerst kluger Mann, von freundlichem Ernste, sehr freigiebig und menschlich gesinnt, der nie gegen Parteien und Gesamtheit etwas versuchte, sondern darauf bedacht war, jedem Wohltaten zu erzeigen und durch seine Freigiebigkeit sich Anhänger unter den Bürgern zu verschaffen. “"

Hier vereinte Cosimo alle Eigenschaften eines guten Politikers, wobei am Ende doch ein Hinweis auf seinen Klientelismus gefunden werden konnte. Dass die herrschenden Schichten in Konfliktzeiten gerne auf Verleumdung und den Vorwurf der Korruption zurückgriffen, lässt sich aus folgendem Zitat herauslesen:

„Daraus entstanden alle übrigen auf Magistrate und Kommissarien gehäuften Beschuldigungen: wirkliche Fehler wurden übermäßig verschrien, erdichtete aufgebracht, und wirkliche und erdichtete von dem meist mit Haß erfüllten Volk geglaubt. “35

Die Beschreibung dieser politischen Praxis, aber auch die Reaktion der Bürger ließ vermuten, dass das Phänomen der Korruption durchaus bekannt und geächtet war.

Eines der einschneidendsten Ereignisse in der Geschichte der Medici, das Attentat auf Lorenzo, wurde auch bei Machiavelli beschrieben. Dieses stellte laut inm ein leuchtendes Beispiel für den Wahnsinn und das Schlechte eines korrupten Menschen dar, weswegen diese Begebenheit oft politisch ausgeschlachtet wurde. Welch großen Einfluss die Medici aufgrund ihrer Patronage und ihres Klientelismus besaßen, beschrieb jene Textstelle:

„Keinen Bürger gab es, der nicht mit oder ohne Waffen nach den Mediceischen Häusern geeilt wäre und in dieser Unordnung sich und seine Habe dargeboten hätte: so groß war die Gunst, in welche die Familie sich durch Klugheit und Liberalität zu setzen gewußt hatte. ${ }^{\text {"36 }}$

33 Shumer, Machiavelli, S. 11-28.

34 Niccolò Machiavelli, Geschichte von Florenz, übs. von Kurt Kluxen, Zürich 1986, S. 262.

35 Ebd., S. 264

36 Ebd., S. 498. 
Obwohl diese Passage eindeutig zu Gunsten der Medici verfasst wurde, war die Auswirkung ihres Klientelnetzwerkes doch deutlich zu sehen. Sicher waren viele Bürger nicht nur aus Gunst zu ihren Häusern geeilt, sondern weil es ihre Pflicht aufgrund der Patronage der Medici war, dieses Attentat zu rächen. Die Auswirkungen und Möglichkeiten eines informellen Netzwerkes, ohne das die Medici sicherlich nicht solch politische Macht hätten erringen können, wurden an diesen Beispielen sehr deutlich aufgezeigt.

In seiner Schrift über die Politik verwies Machiavelli auf die Patronage schon im Bezug auf die Staatengründung. So schrieb er etwa:

„Wer in einem Lande, in dem es viele Mächtige gibt, einen Freistaat errichten will, kann nur dann zum Ziel kommen, wenn er diese vorher alle beseitigt. Wer hingegen in einem Land, in dem der Sinn für Gleichheit stark vertreten ist, eine Monarchie oder eine Gewaltherrschaft errichten will, wird nur zum Ziel kommen, wenn er eine Anzahl ehrgeiziger und unruhiger Köpfe aus der Masse der Gleichberechtigten herausstellt und entsprechend bevorzugt, und zwar nicht nur dem Namen nach, sondern auch tatsächlich, indem er sie mit Machtmitteln und Besitzungen ausstattet und innen Vorrechte jeder Art einräumt. “37

Hier wurde ein System des Klientelismus beschrieben, mit dem ein Herrscher seine Machtposition aufbauen konnte. Machiavelli hatte eine sehr differenzierte Position gegenüber der Korruption inne. Klientelismus und Patronage schienen inn in manchen Fällen nicht wirklich zu stören, Bestechung und Untreue allerdings verurteilte er auf das Schärfste. Dass man einen kleinen Teil der Korruption jedoch tolerieren musste, ließ sich aus seiner These herauslesen.

\section{Schluss}

Zu Beginn der Arbeit wurde die Frage gestellt, ob die Herren der Stadt Florenz Korruption in gewissem Maße tolerieren mussten und ob sich die informelle Politik bedeutend auf die Politik und Regierung auswirkte. Die aufgestellte Hypothese besagte, dass dies sehr wohl der Fall war, da die Herrschaftsstruktur des Stadtstaates Florenz und der Toskana es unabdingbar machten, dass Korruption toleriert werden musste und die informelle Politik sich sehr stark auswirkte. Nun, am Schluss der Arbeit, hat sich diese These bestätigt.

Im Spätmittelalter war der Versuch der Stadt Florenz, die Korruption einzudämmen, noch ersichtlich. Die Regierung versuchte mit Gesetzen und Bestrafungen ihrem Ausgreifen Herr zu werden, schaffte dies aber nicht. Mit dem Aufstieg der Medici wurde die informelle Politik in den Vordergrund gestellt, denn Patronage und Klientelismus waren das Erfolgsrezept dieser Familie. Mit ihrem Leitspruch „Freunde durch Geld“ konnte sie große Erfolge erzielen und loyale Gefolgsleute in wichtigen Schlüsselpositionen der Verwaltung und der Politik einsetzen. Da sie selbst auf dieses Mittel zurückgriff, ließ es sich natürlich nicht vermeiden, dass die Korruption in Florenz Bestand haben würde. 
In den darauffolgenden Jahrhunderten erfolgte ein Wandel dieses Phänomens. Die Korruption wurde mit moralischem Verfall und Niedergang identifiziert, die richterliche Bestechlichkeit wurde stark kritisiert. Auch wenn Florenz in diesem Zusammenhang eine positive Ausnahme darstellte, da viele Gesetze zur Eindämmung von Korruption erlassen wurden, konnte sie doch nicht zur Gänze beseitigt werden. Dies lag unter anderem an besagter Moralisierung der Korruption. Während niedere Schichten von den Strafen abgeschreckt wurden, wurde Korruption als Ausnahme dargestellt und konnte so als tolerabel angesehen werden.

Der Einfluss des Informellen war vor allem am Beispiel der Beziehungen der Florentiner zum Kirchenstaat ersichtlich. Das Zeremoniell bestimmte mitunter die Politik und feine Unterschiede in einer Anrede oder einer Geste konnten gewaltige Auswirkungen haben. Der Fall der Familie Barberini stand dabei warnend für die Gefahren eines solchen Spieles, das katastrophale Folgen haben konnte. Gleichzeitig wurden aber auch die Möglichkeiten und Chancen beschrieben, die mithilfe eines großen Klientelnetzwerkes und Patronagesystems geschaffen werden konnten.

Selbst Niccolò Machiavelli nahm sich dieses Themas an und beschrieb seine Ansichten eines guten Politikers und Herrschers. Florenz diente inm dabei als Exempel, an dem er die Gefahren solchen Handels erläuterte. Zusammenfassend lässt sich sagen, dass Korruption und informelle Politik einen nicht zu unterschätzenden Faktor darstellten. Patronage und Klientelismus waren mächtige politische Waffen, derer die herrschende Klasse sich bedienen konnte. Und selbst, wenn die Korruption ein wenig eingedämmt werden konnte, so verschwand sie niemals zur Gänze.

\section{Literatur und Quellen}

Geltner, Guy/von Berkel, Maaike, Fighting Corruption in Premodernity, East and West: A Literature Review [http://anticorrp.eu/publications/corruption-in-premodernity-east-andwest-a-literature-review/, eingesehen 29.05.2014].

Isenmann, Moritz, Rector est Raptor. Korruption und ihre Bekämpfung in den italienischen Kommunen des späten Mittelalters, in: Karsten, Arne/Thiessen, Hillard von (Hrsg.), Nützliche Netzwerke und korrupte Seilschaften, Göttingen 2006, S. 208-230.

Köchli, Ulrich, Nepoten, Pfründen und Klienten. Die Krise der Familie Barberini nach dem Tod Urbans VIII., in: Karsten, Arne/Thiessen, Hillard von (Hrsg.), Nützliche Netzwerke und korrupte Seilschaften, Göttingen 2006, S. 16-180.

Machiavelli, Niccolò, Gedanken über Politik und Staatsführung, übs. von Rudolf Zorn, Leipzig 1941.

Ders., Geschichte von Florenz, übs. von Kurt Kluxen, Zürich 1986.

Martines, Lauro, Die Verschwörung. Aufstieg und Fall der Medici im Florenz der Renaissance, Darmstadt 2004.

Reinhardt, Volker, Geschichte von Florenz, München 2013. 
Shumer, Sarah, Machiavelli: Republican politics and its corruption, in: Political Theory 7 (1979), H. 1, S. 5-34.

Waquet, Jean-Claude, Corruption. Ethics and power in Florence, 1600-1770, Pennsylvania 1992

Ders., Some considerations on corruption, politics and society in sixteenth and seventeenth century Italy, Waquet, Jean-Claude, Some considerations on corruption, politics and society in sixteenth and seventeenth century Italy, in: Little, Walter/Carbó, Edward Perader (Hrsg.), Political corruption in Europe and Latin America, New York 1996, S. 21-40.

Wieland, Christian, Fürsten, Freunde, Diplomaten. Die römisch-florentinischen Beziehungen unter Paul V. (1605-1621) (Norm und Struktur. Studien zum sozialen Wandel im Mittelalter und der frühen Neuzeit 20), Köln 2004.

Clemens Steinwender ist Student der Bachelorstudien Geschichtswissenschaft und Classica et Orientalia an der Universität Innsbruck. clemens.steinwender@student.uibk. ac. at

\section{Zitation dieses Beitrages}

Clemens Steinwender, Korruption, Ämterkauf und Patronage in Florenz, in: historia. scribere 7 (2015), S. 279-294, [http://historia.scribere.at], 2014-2015, eingesehen 1.3.2015 (=aktuelles Datum).

(c) Creative Commons Licences 3.0 Österreich unter Wahrung der Urheberrechte der Autorlnnen. 
